\title{
On the use of multi-agent systems for the monitoring of industrial systems
}

\author{
Nafissa Rezki ${ }^{1}$ Okba Kazar ${ }^{2}$ Leila Hayet Mouss ${ }^{1} \cdot$ Laid Kahloul $^{2} \cdot$ \\ Djamil Rezki ${ }^{1}$
}

Received: 14 April 2015/Accepted: 2 December 2015/Published online: 15 December 2015

(C) The Author(s) 2015. This article is published with open access at Springerlink.com

\begin{abstract}
The objective of the current paper is to present an intelligent system for complex process monitoring, based on artificial intelligence technologies. This system aims to realize with success all the complex process monitoring tasks that are: detection, diagnosis, identification and reconfiguration. For this purpose, the development of a multi-agent system that combines multiple intelligences such as: multivariate control charts, neural networks, Bayesian networks and expert systems has became a necessity. The proposed system is evaluated in the monitoring of the complex process Tennessee Eastman process.
\end{abstract}

Keywords Multivariate process $\cdot$ Hotelling $T^{2}$ control chart $\cdot$ Multi-agent system $\cdot$ Bayesian network $\cdot$ Neural network

Electronic supplementary material The online version of this article (doi:10.1007/s40092-015-0133-7) contains supplementary material, which is available to authorized users.

Nafissa Rezki

nafissa_rezki@yahoo.fr

Okba Kazar

okbakazar@yahoo.fr

Leila Hayet Mouss

hayet_mouss@yahoo.fr

Laid Kahloul

kahloul2006@yahoo.fr

Djamil Rezki

drezki@yahoo.fr

1 LAP: Laboratory, Industrial Engineering Department, University of Batna, 05000 Batna, Algeria

2 LINFI Laboratory, Computer Science Department, University of Biskra, 07000 Biskra, Algeria

\section{Introduction}

The process monitoring is a critical task in all industrial plant. It can be realized by the use of three principal approaches (Venkatasubramanian et al. 2003): (1) the analytical methods based on mathematics models. These methods compare the real-system outputs to the mathematical model outputs, (2) the methods based on knowledge (Stamatis 2003; Dhillon 2005) that use the human knowledge [risk analysis, failures modes effects and critically analysis (FMECA), decision trees], and (3) the databased methods that focus on statistic development of the process. The last kind of the method uses, generally, the control charts [(Page 1954), cumulative SUM (CUSUM) (Roberts 1959)] or exponentially weighted moving average (EWMA) (Alt et al. 1985) for the fault detection in the industrial process.

Currently, the manufacturing processes become more and more complex and multivariate. In these systems, the operator recuperates a vast data amount to be analysed. The high volume of data and the big number of process variables make the operator task fastidious. To avoid such problems, the data-based methods are more suitable for the process monitoring. The multivariate control charts [Hotelling $T^{2}$ control chart, multivariate CUSUM (MCUSUM), multivariate EWMA (MEWMA)] have been used for the control of multivariate process and have proved their adequacy to reduce the complexity of such process monitoring. Moreover, the monitoring of a multivariate process is a complex task, and it can be devised into four subtasks which are: the detection of abnormal situation, the diagnosis of the faults, the identification of variables that involved in the faults and finally the reconfiguration of the process (Venkatasubramanian et al. 2003). 
Many researches have used the control charts for process monitoring (Yu-Chang et al. 2015; Xia 2015; Ehsan and Sadigh 2014; Vijayababu and Rukmini 2014; Assareh et al. 2013). To identify the variables that make an out-ofcontrol in $T^{2}$, a decomposition of the statistic $T^{2}$ into independent terms has been suggested by Jing et al. (2008). The "MYT approach" has been applied by Mani and Cooper (1999) for the variables identification. The "MYT approach" has a big disadvantage which is the number of $T^{2}$ decompositions. For a process with $p$ variables, the number of decompositions is $p$ !. To reduce this number and to identify the relationship among the variables, the Bayesian networks have been applied for variables identification by Friedman (2000), Li et al. (2006), Li and Shi (2007), Sylvain (2007).

In this paper, we regroup all the tasks of the multivariate process monitoring in one approach. Our contribution is to determine the best combination of multivariate control charts, neural networks, Bayesian networks, expert systems. The result of this research is a multi-agent system that applied to a multivariate process monitoring. This multi-agent system uses: multivariate control chart for abnormal detection, neural network for faults diagnosis, Bayesian network for variables identification and expert system for reconfiguration task.

The rest of this paper is organized as follows: the process monitoring approach is presented in "The proposed multi-agent system" section with the monitoring algorithm. In "Application of the proposed model on the Tennessee Eastman process" section, a case study of simulated Tennessee Eastman process (TEP) (Downs and Vogel 1993) is employed to illustrate the validity of the proposed approach, including the detection by multivariate control charts executor agent (MCCEA), diagnosis by diagnosis artificial neural network agent (DANNA), identification by Identification Bayesian network agent (IBNA) and the reconfiguration by reconfiguration agent (RA). Finally, conclusions and future works are suggested.

\section{The proposed multi-agent system}

The proposed multi-agent system uses a multiple intelligences that are: multivariate control chart, neural network, Bayesian network and expert system in a multi-agent system. The multivariate control charts ( $T^{2}$ control chart, MEWMA ...) can detect successfully the instability of the process, but it cannot diagnosis the fault that appeared in the process and cannot identify the causes of the instability. In this paper, we use an artificial neural network for the faults diagnosis. The neural networks have demonstrated their ability in the classification of similar faults. The neural networks take time in

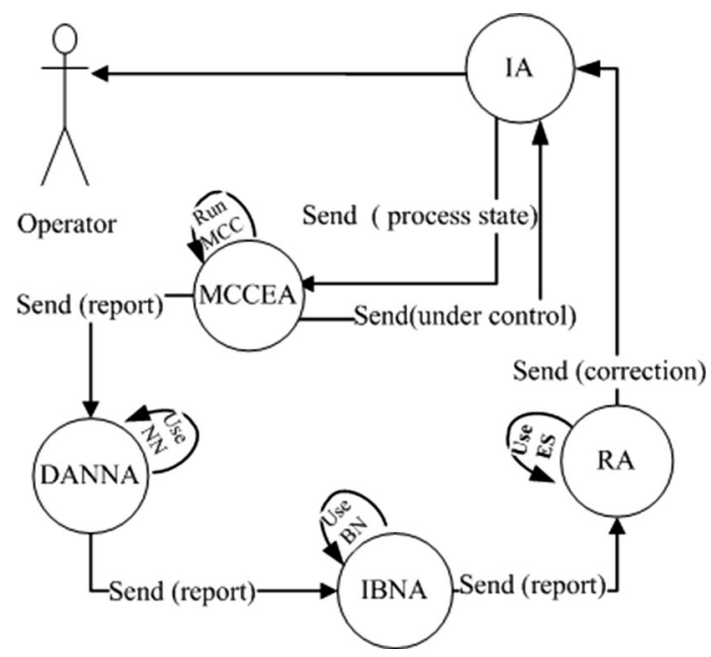

Fig. 1 The agents diagram

the training phase, and then, the classification will be done quickly. After detecting the instability using $T^{2}$ control chart, and the diagnosis using neural network, the Bayesian network proposed by Sylvain (2007) is used in the identification task. To realize a complete monitoring system for multivariate process and simplify the reconfiguration task to the operators that are not specializing in the realm, we developed an expert system that assures the process correction. The following paragraphs will describe each of these used agents. The agent diagram of the proposed approach is shown in Fig. 1. In this diagram, the actual agent types are represented by circles. People that must interact with the system are represented by the unified modelling language (UML) actor symbol.

\section{The interface agent}

The interface agent (IA) is a reactive agent which represents the interface for the human user access; hence, it receives the request from the users (monitoring the process state). Besides this, the IA transforms the agent's responses to the users. The IA receives a request from the user about the process state, and it sends a message to the MCCEA. If the process is under control, the IA will display to the operator the decision of the MCCEA. In the other case, when the process is out of control, the IA waits the response from the RA and displays it to the user.

\section{The multivariate control chart executor agent}

This agent is responsible on the execution of the multivariate control charts $\left[T^{2}\right.$ control chart (Hotelling 1947), multivariate CUSUM (MCUSUM) (Pignatiello and Runger 1990), multivariate EWMA (MEWMA) (Lowry et al. 1992)]. The control charts $\left(T^{2}\right)$ control chart, MEWMA 
and MCUSUM can successfully detect the process instability, but it cannot give any information about the fault that appeared in the process and the variables that are responsible about the process instability. The use of one chart for process monitoring is not sufficient to detect all out of control situation. So, to monitor successively the process, we suggest to use a software agent that can execute simultaneously a set of multivariate control charts and detect easily the process instability. These different control charts are utilized in the design and implementation of the MCCEA.

\section{The diagnosis artificial neural network agent}

We use the neural networks in the diagnosis task because it demonstrated its efficiency in the resolution of classification problem. In addition, the neural networks-after the learning step-has a short response time and a good classification rate. We create a classical multilayer perceptron (MLP), with three layers: (1) the input layer: the number of neurons in this layer is the number of the process parameters, (2) the output layer: in this layer, the number of the neurons represents the number of classes (faults of the process), (3) the hidden layer: it is generally known that the number of neurons in this layer is problematic research. We carried out a set of tests, and we find that the optimal number is equal to: (number of neurons in the input layer + the number of neurons in the output layer)/2. This neural network is used in the implementation of the DANNA. So, in our system the DANNA is responsible for the diagnosis task. When the process is out of control, DANNA receives report from the MCCEA. Its principle objective is to find the fault that appeared in the process. After, it sends a report to the IBNA.

\section{The identification Bayesian network agent}

The IBNA receives report from DANNA about the fault that appeared in the process. It builds a Bayesian net using the causal decomposition algorithm of $T^{2}$ proposed by Sylvain (2007). It finds the variables involved in the fault. This agent simplifies the variable identification in the process. After, it sends report to the RA.

\section{The reconfiguration agent}

For the objective, to regroup all the process monitoring tasks (detection, diagnosis, identification and reconfiguration) in one system, we add the RA which helps the operator to reconfigure the process after its failure. It receives report from the IBNA about the variables that involved in the fault. It must propose a reconfiguration plan to the operator, to maintain the process. Also, it sends its reconfiguration plan to the IA. This agent has been developed using an expert system technology.

\section{The proposed monitoring algorithm}

Start

Get data from data base

Create the MCCEA

MCCEA runs the controls charts

If(MCCEA-decision=stable-process) Then

MCCEA sends report to the IA

Else

Create the DANNA

Create the IBNA

Create RA

DANNA creates the ANN using MLP

IBNA creates the Bayesian net

For (i=1 to number of observations) Do

DANNA gives its diagnosis of the observation $i$

DANNA sends the diagnosis to the IBNA

End For

IBNA receives the diagnosis from DANNA

IBNA uses $\mathrm{BN}$ to find the variables that are out of control

IBNA sends the report to the RA

RA receives report about the variables involved in the fault

RA finds the reconfiguration plan

RA sends report to the IA

IA receives report from RA

End If

End

\section{Application of the proposed model on the Tennessee Eastman process}

\section{Introduction to the Tennessee Eastman process}

The Tennessee Eastman process (TEP) is proposed by Downs and Vogel (1993) to provide a simulated model and to evaluate the monitoring methods of industrial complex process. The process consists of five principal units: a condenser, a separator, a reactor, a compressor and a stripper. Four gaseous reactants (A, C, D and E) and inert B are fed to the reactor. It produces two components ( $\mathrm{G}$ and $\mathrm{H}$ ) and the undesired by-product $\mathrm{F}$. The reaction equations are listed in equation number (1-4). All the reactions are irreversible, exothermic and approximately first order with respect to the reactant concentrations. The reaction rates are expressed as Arrhenius function of temperature. The reaction producing $\mathrm{G}$ has higher activation energy than that 
producing $\mathrm{H}$, thus resulting in more sensitivity to temperature (Fig. 2).

The TEP process proposed by Downs and Vogel (1993) is open loop unstable, and it should be operated under closed loop. In this article, we use this control structure to evaluate the performance of our approach on fault diagnosis. The reactor product stream is cooled through a condenser and fed to a vapour-liquid separator. The vapour exits the separator and recycles to the reactor feed through a compressor. A portion of the recycle stream is purged to prevent the inert and by-product from accumulating. The condensed component from the separator is sent to a stripper, which is used to strip the remaining reactants. Once $\mathrm{G}$ and $\mathrm{H}$ exit the base of the stripper, they are sent to a downstream process which is not included in the diagram. The inert and by-products are finally purged as vapour from vapour-liquid separator. The process provides 41 measured and 12 manipulated variables, denoted as $\mathrm{XMEAS}(1)$ to XMEAS(41) and XMV(1) to XMV(12), respectively. Their brief descriptions and units are listed in
Tables 1 and 2. Fifteen preprogrammed faults IDV(1) to IDV(15) of TEP are given to represent different conditions of the process operation, as listed in Table 3.

$$
\begin{aligned}
& A(g)+C(g)+D(g) \longrightarrow G(l) \\
& A(g)+C(g)+E(g) \longrightarrow H(l) \\
& A(g)+E(g) \longrightarrow F(l) \\
& 3 D(g) \longrightarrow 2 F(l)
\end{aligned}
$$

\section{Simulation and results analyses}

The proposed approach has been implemented using the Java environment Netbeans IDE. Also, we use the agent design platform Java Agent Development framework JADE. To simplify the development of the neural network and Bayesian network with Netbeans, java offers many libraries. Moreover, we use Jess Tab which is a rule engine for the Java platform to produce our rules in the knowledge

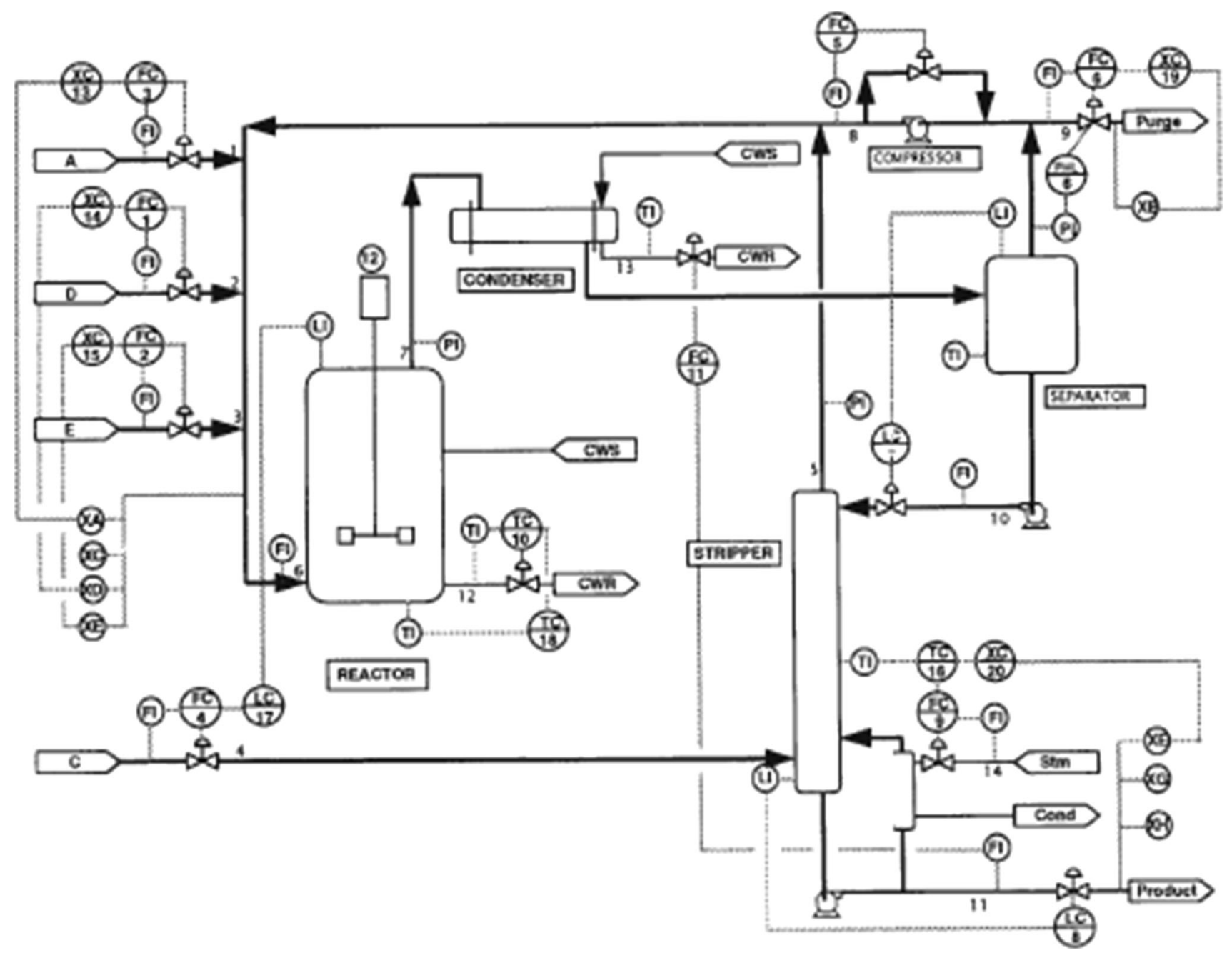

Fig. 2 Tennessee Eastman control problem 
Table 1 Measurement variables in the Tennessee Eastman process

\begin{tabular}{|c|c|c|}
\hline Variable & Description & Units \\
\hline XMEAS(1) & A feed (stream 1) & $\mathrm{kscmh}$ \\
\hline XMEAS(2) & E feed (stream 3) & $\mathrm{kg} / \mathrm{h}$ \\
\hline XMEAS(4) & Total feed (stream 4) & $\mathrm{kg} / \mathrm{h}$ \\
\hline XMEAS(5) & Recycle flow (stream 8) & kscmh \\
\hline XMEAS(6) & Recycle flow (stream 6) & kscmh \\
\hline XMEAS(7) & Reactor pressure & $\mathrm{kPa}$ gauge \\
\hline XMEAS(8) & Reactor level & $\%$ \\
\hline XMEAS(9) & Reactor temperature & $\mathrm{C}^{\circ}$ \\
\hline XMEAS(10) & Purge rate (stream 9) & $\mathrm{kscmh}$ \\
\hline XMEAS(11) & Product sep temp & $\mathrm{C}^{\circ}$ \\
\hline XMEAS(12) & Product sep level & $\%$ \\
\hline XMEAS(13) & Prod sep pressure & $\mathrm{kPa}$ gauge \\
\hline XMEAS(14) & Prod sep underflow (stream 10) & $\mathrm{m}^{3} / \mathrm{h}$ \\
\hline XMEAS(15) & Stripper level & $\%$ \\
\hline XMEAS(16) & Stripper pressure & $\mathrm{kPa}$ gauge \\
\hline XMEAS(17) & Stripper underflow (stream 11) & $\mathrm{m}^{3} / \mathrm{h}$ \\
\hline XMEAS(18) & Stripper temperature & $\mathrm{C}^{\circ}$ \\
\hline XMEAS(19) & Stripper steam flow & $\mathrm{kg} / \mathrm{h}$ \\
\hline XMEAS(20) & Compressor work & $\mathrm{kW}$ \\
\hline XMEAS(21) & Reactor cooling water outlet temp & $\mathrm{C}^{\circ}$ \\
\hline XMEAS(22) & Separator cooling water outlet temp & $\mathrm{C}^{\circ}$ \\
\hline Variable & Description & Stream \\
\hline XMEAS(23) & Component A & 6 \\
\hline XMEAS(24) & Component B & 6 \\
\hline XMEAS(25) & Component $\mathrm{C}$ & 6 \\
\hline XMEAS(26) & Component D & 6 \\
\hline XMEAS(27) & Component E & 6 \\
\hline XMEAS(28) & Component $\mathrm{F}$ & 6 \\
\hline XMEAS(29) & Component A & 9 \\
\hline XMEAS(30) & Component B & 9 \\
\hline XMEAS(31) & Component $\mathrm{C}$ & 9 \\
\hline XMEAS(32) & Component D & 9 \\
\hline XMEAS(33) & Component E & 9 \\
\hline XMEAS(34) & Component $\mathrm{F}$ & 9 \\
\hline XMEAS(35) & Component $\mathrm{G}$ & 9 \\
\hline XMEAS(36) & Component $\mathrm{H}$ & 9 \\
\hline XMEAS(37) & Component D & 11 \\
\hline XMEAS(38) & Component E & 11 \\
\hline XMEAS(39) & Component $\mathrm{F}$ & 11 \\
\hline XMEAS(40) & Component $\mathrm{G}$ & 11 \\
\hline XMEAS(41) & Component $\mathrm{H}$ & 11 \\
\hline
\end{tabular}

base. In this work, we use FIPA Agent Communication specifications that deal with Agent Communication Language (ACL) messages, message exchange interaction protocols and content language representations.
Table 2 Manipulated variables in the Tennessee Eastman process

\begin{tabular}{ll}
\hline Variable & Description \\
\hline XMV(1) & D feed flow (stream 2) \\
XMV(2) & E feed flow (stream 3) \\
XMV(3) & A feed flow (stream 1) \\
XMV(4) & Total feed flow (stream 4) \\
XMV(5) & Compressor recycle valve \\
XMV(6) & Purge valve (stream 9) \\
XMV(7) & Separator pot liquid flow (stream 10) \\
XMV(8) & Stripper liquid product flow (stream 11) \\
XMV(9) & Stripper steam valve \\
XMV(10) & Reactor cooling water flow \\
XMV(11) & Condenser cooling water flow \\
XMV(12) & Agitator speed \\
\hline
\end{tabular}

In this section, we evaluate the performances of the proposed approach on concrete example which is the TEP process. The used data represent 480 observations training for each fault and 800 tests for each faults, in addition to the normal period. The observations of training have been obtained with the simulation of each fault in a period of $24 \mathrm{~h}$; moreover, the observations of the test set have been obtained in a period of $40 \mathrm{~h}$. Variables are sampled every 3 min.

\section{- The Detection}

All the persons that are worked on TEP take rate to obtain wrong alarm equal to $0.01 \%$. In this work, we use the $T^{2}$ control chart for instability detection. A performance of detection system is evaluated by calculating its reliability (Kononenko 1991). The detection reliability is defined as: (the number of obtained alerts in the test period/the total number of sample in the period test).

The MCCEA runs the $T^{2}$ control chart; if it detects an abnormal process state, it sends message to the DANNA. The detection reliability obtained in this work is the same that been obtained by Sylvain (2007). Figure 3 shows the detection reliability of MCCEA, and some faults are easily detectable [IDV (1), IDV (2), IDV (4), IDV (5), IDV (6), IDV (7), IDV (8), IDV (10), IDV (12), IDV (14)]. But other faults are difficult to detect [(IDV (3), IDV (9) and IDV (15)]. The last faults [(IDV (3), IDV (9), IDV (15)] are very identical. So the use of one chart (in this work, we use the $T^{2}$ control chart) is not sufficient. The run of many control charts simultaneously will augment the reliability of detection.

- The Diagnosis

This task is realized by the DANNA. When it receives message from the MCCEA that the process is not stable, it creates the neural network using MLP, for the purpose 
Table 3 The known faults of the Tennessee Eastman process

\begin{tabular}{lll}
\hline Variable & Description & Type \\
\hline IDV(1) & A/C feed ratio, B composition constant (stream 4) & Step \\
IDV(2) & B composition, A/C ratio constant (Stream 4) & Step \\
IDV(3) & D feed temperature (stream 2) & Step \\
IDV(4) & Reactor cooling water inlet temperature & Step \\
IDV(5) & Condenser cooling water inlet temperature & Step \\
IDV(6) & A feed loss (stream 1) Step & Step \\
IDV(7) & C header pressure loss-reduced availability (stream 4) & Step \\
IDV(8) & A, B, C feed composition (stream 4) & Random variation \\
IDV(9) & D feed temperature (stream 2) & Random variation \\
IDV(10) & C feed temperature (stream 4) & Random variation \\
IDV(11) & Reactor cooling water inlet temperature & Random variation \\
IDV(12) & Condenser cooling water inlet temperature & Random variation \\
IDV(13) & Reaction kinetics & Slow drift \\
IDV(14) & Reactor cooling water valve & Sticking \\
IDV(15) & Condenser cooling water valve & Sticking \\
\hline
\end{tabular}

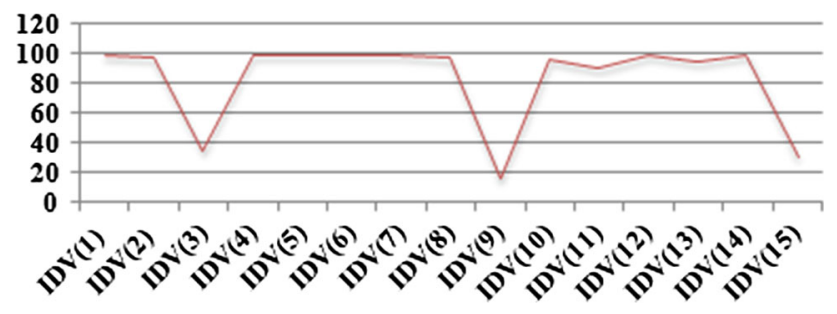

Fig. 3 The detection reliability

to find the fault that appeared in the process. In the next paragraph, we will show the diagnosis obtained by the DANNA and we will evaluate the acquired results to the result of other classifiers proposed in the literature.

\section{Diagnosis of the known faults in the Tennessee Eastman problem}

We have done the diagnosis of all the faults, i.e. IDV (1) to IDV (15) in TEP, as shown in Fig. 4. The used neural network is a MLP of three layers:

- The input layer contains 53 neurons that represent the process parameters,

- The hidden layer contains 34 neurons (number of neurons in input layer + number neurons in output layer/2),

- The output layer contains 15 neurons that represent the process faults.

Table 4 represents a comparison between the diagnosis realized by DANNA, and some other approaches proposed to the TEP faults diagnosis. Sylvain (2007) used Bayesian network for classification; however, the PC1DARMF ( $\mathrm{Li}$ and Xiao 2011) is a supervised pattern classification method which uses one-dimensional adaptive rank-order morphological filter.

\section{Diagnosis of IDV (4), IDV(9), IDV(11) in TEP}

The most difficult faults to be classified in the TEP are: IDV (4), IDV (9) and IDV (15). The created neural network composed by 53 neurons (TEP parameters) in the input layer, 28 neurons in the hidden layer and 3 neurons in the output layer. Table 5 presents the rate of correct classification of the faults IDV (4), IDV (9) and IDV (15) of the TEP. It is a comparison between the DANNA diagnosis and the approach which proposed by El-Ferchichi (2013).

- The Identification

The IBNA is the responsible on the realization of the identification task using Bayesian net. It receives a report about the fault that appeared in the process from DANNA. To develop the Bayesian network, Sylvain (2007) used the causal decomposition of $T^{2}$. Figure 4 presents the Bayesian network that is created in the normal functionality of process. We take rate of false alarm $=0.005$. The IBNA takes the observation that represents the fault, and then, it finds the variables that are involved in the fault. The variables involved in the fault have probability value under 0.995 . We take the case of the observation 240 of IDV (5) that is classified as an IDV (4). The IBNA detects two variables that have a probability value under 0.995 . The two variables are (XMV11) and (XMEAS21). The IBNA sends the variable identification to the RA.

- The Reconfiguration

The RA receives report from IBNA which contains the identification of the variables that cause the process instability. In our example, the identified variables are (XMV11) and (XMEAS21). The RA finds that: the variable (XMV11) represents the liquid cooling flow to the condenser, whereas the variable (XMEAS21) 


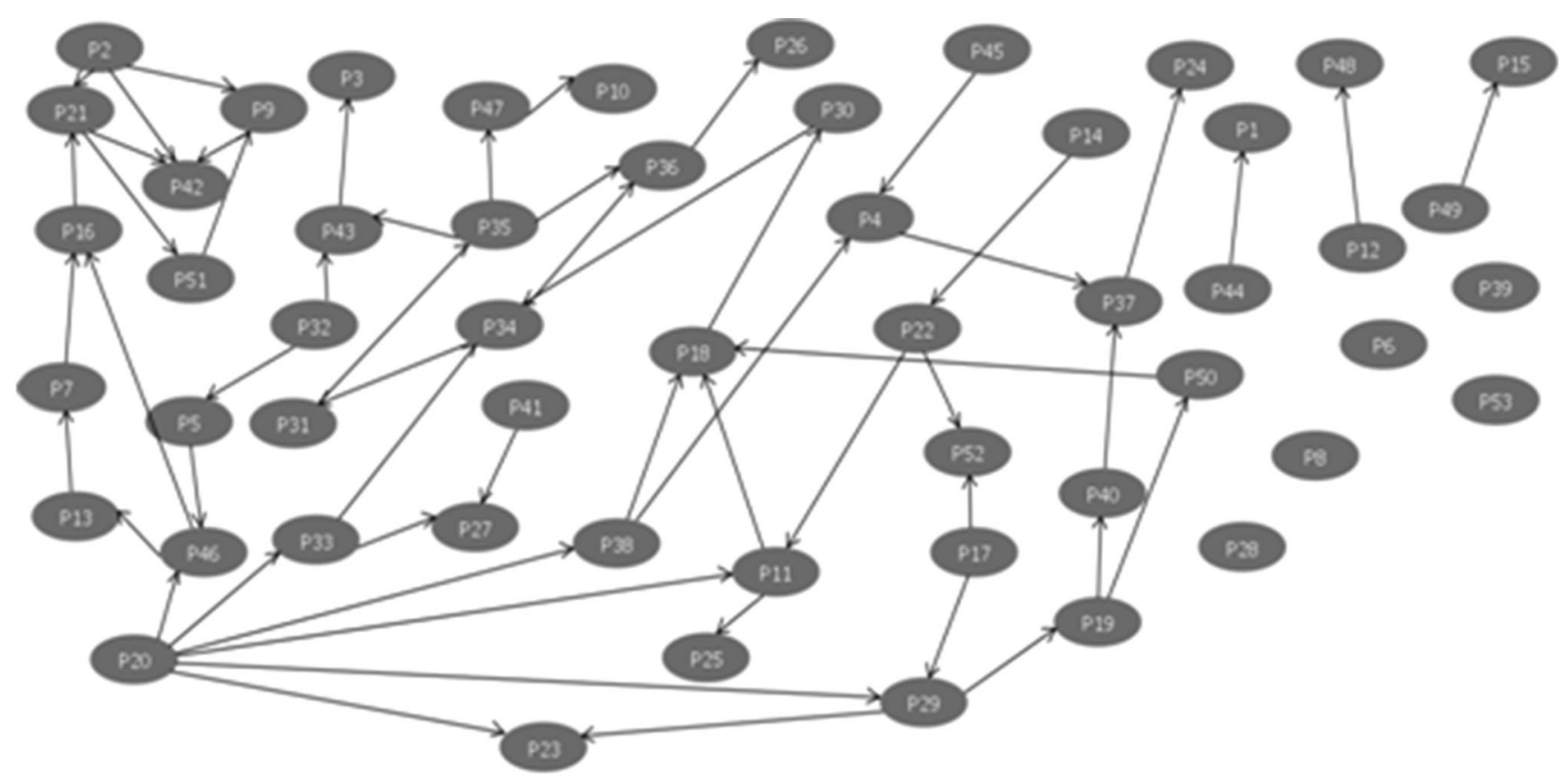

Fig. 4 The Bayesian network that used in the development of IBNA

Table 4 Classification rate of the known 15 faults in TEP

\begin{tabular}{lclc}
\hline Faults & DANNA $(\%)$ & Sylvain $(2007)(\%)$ & PC1DARMF Li and Xiao (2011) (\%) \\
\hline IDV(1) & 97.01 & 97.5 & 30 \\
IDV(2) & 95.34 & 98.125 & 95 \\
IDV(3) & 82.10 & 22 & 0.00 \\
IDV(4) & 97.34 & 82.375 & 25 \\
IDV(5) & 96.67 & 98 & 100 \\
IDV(6) & 100 & 100 & 65 \\
IDV(7) & 97.67 & 100 & 0.00 \\
IDV(8) & 100 & 97 & 5 \\
IDV(9) & 79.06 & 22.625 & 0.00 \\
IDV(10) & 71.42 & 86.875 & 15 \\
IDV(11) & 69.1 & 75.5 & 0.00 \\
IDV(12) & 96.67 & 98.25 & 5 \\
IDV(13) & 100 & 76.125 & 5 \\
IDV(14) & 93.02 & 98.75 & 5 \\
IDV(15) & 92.69 & 23.5 & 0.00 \\
\hline
\end{tabular}

Table 5 Classification rate of IDV (4), IDV (9) and IDV (15) in TEP

\begin{tabular}{lll}
\hline Faults & DANNA (\%) & El-Ferchichi (2013) (\%) \\
\hline IDV(4) & 97.34 & 67.37 \\
IDV(9) & 100 & 66.25 \\
IDV(15) & 100 & 33.75 \\
\hline
\end{tabular}

represents the cooling liquid temperature at the reactor outlet. In conclusion, these two variables involved in the fault IDV (5), so the fault that appeared in the process is the IDV (5) and not the IDV (4). It proposes the reconfiguration plan to the operator. The development of this agent requires knowledge of an expert human, which we will use to find the ideal reconfiguration plan.

\section{Conclusion}

An approach with several intelligences has been proposed in this paper for multivariate process monitoring. In this approach, we use the perfect tool for the realization of each task in a complex process monitoring. We use the multivariate control charts in the detection task. We utilize the 
artificial neural network classifier with MLP algorithm in the diagnosis task. For the identification task, we exploit the Bayesian network that has been proposed by Sylvain (2007). Moreover, to help the operators that are not specializing in realm, to realize the correction actions of the process, we suggest developing an expert system for reconfiguration task. To facilitate the use of the proposed approach with high efficiency, we integrate the different proposed subsystem (detection, diagnosis, identification and reconfiguration) in one system that is multi-agent system. The proposed model has been evaluated on a multivariate process (Tennessee Eastman process).

From the simulation results, we find that the proposed classifier gives a good result compared with some works applied on Tennessee Eastman process. In addition, the proposed approach gives good results for each task in the process monitoring. In the case study, we have seen that some faults are difficult for detecting; our future works will concentrate on the development of the detection task. The developed reconfiguration agent realizes the reconfiguration tasks for known faults, and we will focus also on adding the reconfiguration plan in case when a new fault appear in the process.

Acknowledgments The authors would like to express their sincere appreciation for all support provided.

Open Access This article is distributed under the terms of the Creative Commons Attribution 4.0 International License (http://crea tivecommons.org/licenses/by/4.0/), which permits unrestricted use, distribution, and reproduction in any medium, provided you give appropriate credit to the original author(s) and the source, provide a link to the Creative Commons license, and indicate if changes were made.

\section{References}

Alt FB, Kotz NL, Johnson C (1985) R, Read multivariate quality control. Encycl Stat Sci 6:111-122

Assareh H, Noorossana R, Mengersen K-L (2013) Bayesian change point estimation in poisson-based control charts. J Ind Eng Int 9:32

Dhillon B (2005) Reliability, quality, and safety for engineers. CRC Press, Boca Raton

Downs JJ, Vogel EF (1993) A plant-wide industrial process control problem. Comput Chem Eng 17(3):245-255
Ehsan B, Sadigh R (2014) Economic design of Hotellings $T^{2}$ control chart on the presence of fixed sampling rate and exponentially assignable causes. J Ind Eng Int 10:229-238

El-Ferchichi S (2013) Ph.d. Thesis: selection and extraction of attributes for classification problem, National School of engineering of Tunis

Friedman N (2000) Using Bayesian networks to analyze expression data. J Comput Biol 7(3-4):601-620

Hotelling H (1947) Multivariate quality control. In: Eisenhart C, Hastay MW, Wallis WA (eds) Techniques of statistical analysis. McGraw-Hill, New York, pp 111-184

Jing L, Jionghua J, Jianjun S (2008) Causation-based $\mathrm{T}^{2}$ decomposition for multivariate process monitoring and diagnosis. J Qual Technol 40(1):1-13

Kononenko I (1991) Semi-naive bayesian classier, proceeding of the European working session on learning on machine learning, pp 206-219

Li H, Xiao D (2011) Fault diagnosis of Tennessee Eastman process using signal geometry matching technique. J Adv Signal Proc (83). doi:10.1186/1687-6180-2011-83

Li J, Shi J, Satz D (2006) Modelling and analysis of disease and risk factors through learning Bayesian network from observational data, Technical report

Li J, Shi J (2007) Knowledge discovery from observational data for process control using causal Bayesian networks. IIE Trans 39(6):681-690

Lowry CA, Woodall WH, Champ CW, Rigdon SE (1992) A multivariate exponentially weighted moving average control chart. Technometrics 34(1):46-53

Mani S, Cooper GF (1999) A study in causal discovery from population-based infant birth and death records. In: Proceeding of the AMIA annual fall symposium. Philadelphia, pp 315-319

Page ES (1954) Continuous inspection schemes. Biometrika 41:100115

Pignatiello J, Runger G (1990) Comparisons of multivariate cusum charts. J Qual Technol 22(3):173186

Roberts SW (1959) Control chart tests based on geometric moving averages. Technometrics 1(3):239250

Stamatis DH (2003) Failure mode and effect analysis: FMEA from theory to execution. ASQ Quality Press, Milwaukee

Sylvain V (2007) Diagnostic et surveillance des processus complexe par rseaux baysiens.(Diagnosis and monitoring of complex process using bayesian networks), doctoral thesis, University of Angers, French

Venkatasubramanian V, Rengaswamy R, Yin K, Kavuri S (2003) A review of process fault detection and diagnosis, part I: quantitative model-based methods. Comput Chem Eng 27(3):293-311

Vijayababu V, Rukmini V-K (2014) Economic design of x-bar control charts considering process shift distributions. J Ind Eng Int 10:163171

Xia P (2015) Horizontal cumulative variance chart: A quality control scheme monitoring shifts in process variation. Int J Ind Syst Eng

Yu-Chang L, Chao-Yu C, Chung-Ho C (2015) Robustness of the EWMA median control chart to non-normality. Int J Ind Syst Eng 